\title{
Relationship Between Cardiac 123I-Metaiodobenzylguanidine Imaging and the Transcardiac Gradient of Neurohumoral Factors in Patients With Dilated Cardiomyopathy
}

\author{
Toshiki Matsui, MD; Takayoshi Tsutamoto, MD; Masahiko Kinoshita, MD
}

\begin{abstract}
Cardiac sympathetic nervous function is altered in congestive heart failure (CHF) and the uptake and washout rate of cardiac ${ }^{123}$ I-metaiodobenzylguanidine (MIBG) are useful markers for evaluating the severity of it. To assess what parameters predict decreased uptake or increased washout rate of MIBG, the concentrations of neurohumoral factor in both the aorta (Ao) and coronary sinus (CS) were measured, as well as hemodynamic parameters by catheterization, in patients with dilated cardiomyopathy (DCM). MIBG imaging was performed within 1 week of cardiac catheterization. Regarding MIBG parameters, the correlation with the transcardiac gradient of norepinephrine (NE), brain natriuretic peptide (BNP) and hemodynamics was investigated. Stepwise multivariate regression analysis was used to determine which variables closely correlated with cardiac MIBG parameters. There was a significant increase in the NE level between the Ao $(446 \mathrm{pg} / \mathrm{ml})$ and the CS $(727 \mathrm{pg} / \mathrm{ml})$. According to stepwise multivariate regression analysis, the heart/mediastinum (H/M) ratio independently correlated with the transcardiac gradient of BNP $(r=-0.480, p<0.01)$, and the washout rate independently correlated with the transcardiac gradient of NE $(\mathrm{r}=0.481, \mathrm{p}<0.01)$. These findings indicate that the $\mathrm{H} / \mathrm{M}$ ratio may reflect the transcardiac gradient of BNP, which implies the degree of left ventricular dysfunction and/or damage and the washout rate may reflect altered cardiac sympathetic nerve terminal in DCM patients with CHF, suggesting that both the $\mathrm{H} / \mathrm{M}$ ratio and washout rate provide important information about the failing ventricle. (Jpn Circ J 2001; 65: 1041-1046)
\end{abstract}

Key Words: Dilated cardiomyopathy; Metaiodobenzylguanidine (MIBG); Neurohumoral factors; Transcardiac gradient

1 ${ }^{23}$ I-metaiodobenzylguanidine (MIBG) and norepinephrine (NE) are thought to share the same uptake storage (uptake-1 system, uptake-2 system and vesicular uptake) and release mechanisms in the adrenergic nerve terminals!, ${ }^{1}$ However, MIBG is not metabolized by catechol-o-methyltransferase and monoamine oxidase and does not affect adrenergic membrane receptors?2 so MIBG scintigraphy can noninvasively assess sympathetic nervous distribution and function in vivo! Previous studies have reported that the heart/mediastinum $(\mathrm{H} / \mathrm{M})$ uptake ratio and washout rate of cardiac MIBG are prognostic indicators in congestive heart failure (CHF) and sensitive markers that reflect the response to therapies in patients with $\mathrm{CHF}^{3-9}$ Although MIBG parameters correlate with the left ventricular ejection fraction (LVEF) or plasma NE, $3,10,11$ which is reflected not only by cardiac sympathetic nerve activity, but also by systemic sympathetic nerve activity, it remains unknown which markers closely influence MIBG parameters (H/M ratio and washout rate) in patients with CHF.

Increased levels of multiple neurohumoral factors have been found in patients with CHF, and high plasma levels of $\mathrm{NE}$, renin, endothelin-1, atrial natriuretic peptide, and brain natriuretic peptide (BNP) have been reported to be significant prognostic predictors, ${ }^{12-18}$ suggesting an important role

(Received June 25, 2001; revised manuscript received August 30, 2001; accepted September 14, 2001)

First Department of Internal Medicine, Shiga University of Medical Science, Otsu, Japan

Mailing address: Takayoshi Tsutamoto, MD, First Department of Internal Medicine, Shiga University of Medical Science, Tsukinowa, Seta, Otsu 520-2152, Japan. E-mail: tutamoto@ belle.shiga-med.ac.jp of neurohumoral activation in the pathogenesis of CHF. In particular, the plasma level of BNP, mainly from the ventricle ${ }^{19}$ has been reported to be a sensitive and major prognostic marker!7,18,20,21 However, to our knowledge, there are no reports comparing MIBG parameters and the transcardiac differences of NE and BNP, which would provide more direct information about the failing heart than those of peripheral blood samples in patients with CHF.

Therefore, this study evaluated the relationships between MIBG parameters ( $\mathrm{H} / \mathrm{M}$ ratio and washout rate) and the hemodynamics and transcardiac gradient of neurohumoral factors (NE and BNP) in CHF patients with dilated cardiomyopathy (DCM).

\section{Methods}

\section{Patients}

Forty-two consecutive DCM patients (30 men, 12 women; mean age, 56 years) with left ventricular dysfunction (LVEF $\leq 45 \%)$ were included in the study. All patients underwent heart catheterization and endomyocardial biopsy. The DCM were diagnosed by clinical history, ECG, chest roentgenogram, echocardiogram, heart catheterization and endomyocardial biopsy. Patients with valvular disease, ischemic cardiomyopathy, chronic renal failure, diabetes mellitus, and autonomic nervous system disorder were excluded. Twenty-nine patients were classified in New York Heart Association (NYHA) functional class II as mild $\mathrm{CHF}$, and the other 13 in class III or IV were classified as severe CHF. At the time of the study, the 42 patients were receiving the following treatments: digitalis $(n=28)$, diuret- 
Table 1 Clinical Characteristics, Hemodynamics and MIBG Parameters

\begin{tabular}{|c|c|c|}
\hline & Mild CHF $(n=29)$ & Severe CHF $(n=13)$ \\
\hline Age (years) & $55 \pm 2$ & $60 \pm 3$ \\
\hline Gender $(M / F)$ & $19 / 10$ & $11 / 2$ \\
\hline NYHA (functional class) & $2.00 \pm 0.00$ & $3.23 \pm 0.17 *$ \\
\hline$H R($ beats/min $)$ & $74 \pm 3$ & $83 \pm 8$ \\
\hline Systolic BP (mmHg) & $115 \pm 5$ & $101 \pm 5$ \\
\hline$C I\left(L \cdot \mathrm{min}^{-1} \cdot \mathrm{mm}^{-2}\right)$ & $2.60 \pm 0.09$ & $2.42 \pm 0.13$ \\
\hline$P C W P(m m H g)$ & $10.2 \pm 1.2$ & $20.8 \pm 2.1^{*}$ \\
\hline $\operatorname{LVEF}(\%)$ & $34.1 \pm 1.6$ & $27.4 \pm 1.9 * *$ \\
\hline CTR $(\%)$ & $56.9 \pm 1.3$ & $60.1 \pm 2.4$ \\
\hline$H / M$ ratio (delayed) & $1.89 \pm 0.05$ & $1.69 \pm 0.07 * *$ \\
\hline Washout rate $(\%)$ & $39.3 \pm 1.9$ & $46.8 \pm 3.2 * *$ \\
\hline \multicolumn{3}{|l|}{ Treatments } \\
\hline Diuretics & $25(83 \%)$ & $13(100 \%)$ \\
\hline Digitalis & $20(67 \%)$ & $8(62 \%)$ \\
\hline Vasodilators & $3(10 \%)$ & $2(15 \%)$ \\
\hline$\beta$-blockers & $7(23 \%)$ & $4(31 \%)$ \\
\hline ACE-inhibitors & $19(63 \%)$ & $11(85 \%)$ \\
\hline
\end{tabular}

NYHA, New York Heart Association; HR, heart rate; BP, blood pressure; CI, cardiac index; PCWP, pulmonary capillary wedge pressure; LVEF, left ventricular ejection fraction; CTR, cardiothoratic ratio; H/M, heart to mediastinum ratio; ACE, angiotensinconverting enzyme. ${ }^{*} p<0.01$ vs mild $C H F,{ }^{* *} p<0.05$ vs mild $C H F$.

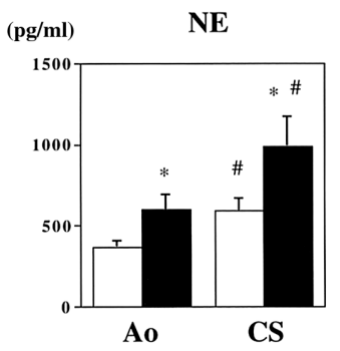

ics $(n=38)$, angiotensin-converting enzyme inhibitors $(n=30), \beta$-blockers $(n=11)$, and vasodilators $(n=5)$. Informed consent was obtained from all subjects for participation in the study according to a protocol approved by the Committee on Human Investigation at the institution.

\section{Cardiac Catheterization}

After $20 \mathrm{~min}$ of bed-rest in a supine position and premedication with diazepam (5 mg orally), a 7Fr Swan-Ganz catheter was inserted into the femoral vein, and the pulmonary capillary wedge pressure (PCWP) and cardiac output were measured. After right heart catheterization, a 6Fr catheter (Goodman, Tokyo, Japan) was placed in the coronary sinus (CS) and blood samples were taken. Using a pigtail catheter inserted through the femoral artery, blood samples were taken from the ascending aorta (Ao), after which the catheter was inserted into the left ventricle, and left ventriculography was performed, from which the LVEF was determined. Finally, coronary arteriography was performed.

\section{Measurement of Plasma BNP and NE}

Blood samples for the assay of BNP levels were transferred to chilled disposable tubes containing aprotinin (500 kallikrein inactivator $\mathrm{U} / \mathrm{ml}$ ) and ethylenediaminetetraacetic acid (EDTA; $1 \mathrm{mg} / \mathrm{ml}$ ), and those for assay of plasma NE levels were transferred to chilled disposable tubes containing EDTA $(1 \mathrm{mg} / \mathrm{ml})$. The blood samples were immediately placed on ice and centrifuged at $4^{\circ} \mathrm{C}$, and aliquots of plasma were immediately stored at $-30^{\circ} \mathrm{C}$ until assayed.

Plasma BNP levels were measured with a specific immunoradiometric assay for human BNP (Shionogi BNP Kit, Osaka Japan $)^{22}$ and plasma NE levels were measured by high-performance liquid chromatography as reported previously. 22

\section{MIBG Imaging}

Each patient was administered $111 \mathrm{MBq}$ of ${ }^{123} \mathrm{I}-\mathrm{MIBG}$ by intravenous injection and myocardial images were acquired using a 3-head gamma camera equipped with a low energy collimator (GCA 9300A, Toshiba, Japan). A 5min static acquisition was performed on an anterior view of the chest, $15 \mathrm{~min}$ (early image) and $3 \mathrm{~h}$ (delayed image) after isotope injection. Energy discrimination was provided by a $20 \%$ window centered at $159 \mathrm{KeV}$. Left ventricular activity was recorded with manually drawn a region of interest (ROI). Another $20 \pm 20$ pixel ROI was placed over the upper mediastinal area $(\mathrm{M})$ and the heart-to-mediastinum ratio $(\mathrm{H} / \mathrm{M})$ was calculated to quantify cardiac MIBG uptake by the mean counts. MIBG washout rate was defined as the percentage change in cardiac activity $(\mathrm{H})$ between the early and delayed images within the left ventricular area: $\{[(\mathrm{H})-(\mathrm{M})]$ early $-[(\mathrm{H})-(\mathrm{M})]$ delayed $\}$ / $[(\mathrm{H})-(\mathrm{M})]$ early $\times 100(\%)$.

\section{Statistical Analysis}

All results are expressed as mean \pm SE. Statistical comparisons were made with Student's paired t test. Categorical data were compared using the chi-square test. Univariate and stepwise multivariate regression analyses were used to assess the relationship between different variables and cardiac MIBG parameters. A value of $\mathrm{p}<0.05$ was consid- 
Table 2 Univariate and Multivariate Linear Models of Clinical, Hemodynamic Parameters and Plasma Neurohumoral Factors for the H/M Ratio

\begin{tabular}{|c|c|c|c|c|}
\hline \multirow{3}{*}{ Variable } & \multicolumn{4}{|c|}{ H/M ratio } \\
\hline & \multicolumn{2}{|c|}{ Univariate } & \multicolumn{2}{|c|}{ Multivariate } \\
\hline & Correlation coefficient & $p$ value & $\beta$-coefficient & p value \\
\hline Age & -0.033 & 0.8366 & & \\
\hline Gender $($ male $=1)$ & -0.074 & 0.6407 & & \\
\hline$A o-B N P$ & -0.343 & 0.0262 & & \\
\hline$C S-B N P$ & -0.466 & 0.0019 & & \\
\hline$(C S-A o) B N P$ & -0.480 & 0.0013 & -0.480 & 0.0013 \\
\hline$A o-N E$ & -0.209 & 0.1851 & & \\
\hline$C S-N E$ & -0.313 & 0.0438 & & \\
\hline$(C S-A o) N E$ & -0.325 & 0.0356 & & \\
\hline LVEF & 0.368 & 0.0165 & & \\
\hline$P C W P$ & -0.341 & 0.0270 & & \\
\hline CTR & -0.274 & 0.0787 & & \\
\hline
\end{tabular}

Ao, aorta; CS, coronary sinus; (CS-Ao), transcardiac gradient; BNP, brain natriuretic peptide; NE, norepinephrine; LVEF, left ventricular ejection fraction; PCWP, pulmonary capillary wedge pressure; CTR, cardiothoracic ratio.

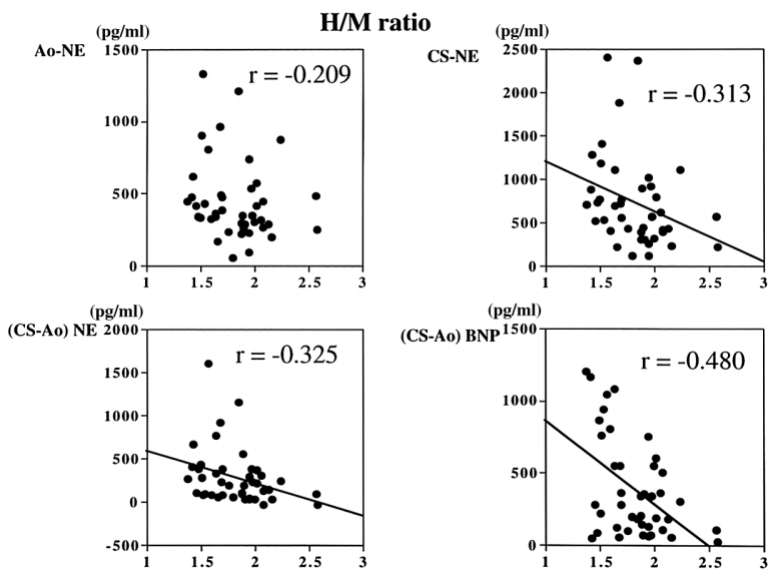

Fig 2. Correlations between $\mathrm{H} / \mathrm{M}$ ratio and neurohumoral factors in patients with dilated cardiomyopathy. ered significant.

\section{Results}

Clinical, Hemodynamics and Scintigraphic Data of Patients

The mean NYHA functional class was 2.4 (NYHA II= 29, III $=9, I V=4)$. The mean LVEF was $32.0 \pm 1.3 \%$. In the scintigraphic data, the mean $\mathrm{H} / \mathrm{M}$ (delayed) was $1.83 \pm 0.04$ and the mean washout rate was $41.6 \pm 1.7 \%$. Table 1 shows age, gender, NYHA functional class, cardiac catheterization data and the mean values of the cardiac MIBG parameters divided by mild (NYHA II) and severe CHF (NYHA III-IV). PCWP and washout rate were significantly increased and LVEF and H/M(delayed) were significantly decreased in patients with severe CHF compared with those with mild CHF.

\section{Plasma Neurohumoral Factors in Ao and CS (Fig 1)}

The plasma levels of NE and BNP in Ao and CS were significantly increased in patients with severe CHF compared with mild CHF. There was a highly significant step-up of the plasma NE level between the Ao and CS in both mild and severe CHF patients with DCM. There was also a significant step-up of the plasma BNP level between the Ao and CS in both mild CHF and severe CHF patients with DCM.

\section{Correlation of the H/M in MIBG With Hemodynamic Parameters and Neurohumoral Factors}

Correlations between the $\mathrm{H} / \mathrm{M}$ (delayed) and age, gender, hemodynamics and neurohumoral factors are shown in Table 2. The H/M(delayed) had a significant positive correlation with LVEF and significant negative correlations with Ao-BNP levels, CS-BNP levels, transcardiac gradient of the BNP, CS-NE levels, transcardiac gradient of the NE (Fig 2), and PCWP. However, H/M(delayed) did not show any correlation with age, gender, Ao-NE levels or cardiothoracic ratio (CTR). According to stepwise multivariate regression analysis among the 11 variables listed in Table 2 , only the transcardiac gradient of BNP was an independent predictor of $\mathrm{H} / \mathrm{M}$ (delayed) in patients with $\mathrm{DCM}$.

\section{Correlation of the Washout Rate in MIBG With Hemo-} dynamic Parameters and Neurohumoral Factors

The correlations between the washout rate and age, gender, hemodynamics and neurohumoral factors are shown in Table 3. The washout rate had significant positive correlations with CS-BNP levels, transcardiac gradient of the BNP, Ao-NE levels (Fig 3), CS-NE levels and transcardiac gradient of the NE and a significant negative correlation with LVEF.

According to stepwise multivariate regression analysis among the 11 variables listed in Table 3, only the transcardiac gradient of NE was an independent predictor of 
Table 3 Univariate and Multivariate Linear Models of Clinical, Hemodynamic Parameters and Plasma Neurohumoral Factors for the MIBG Washout Rate

\begin{tabular}{|c|c|c|c|c|}
\hline \multirow{3}{*}{ Variable } & \multicolumn{4}{|c|}{ Washout rate } \\
\hline & \multicolumn{2}{|c|}{ Univariate } & \multicolumn{2}{|c|}{ Multivariate } \\
\hline & Correlation coefficient & $p$ value & $\beta$-coefficient & $p$ value \\
\hline Age & 0.074 & 0.6407 & & \\
\hline Gender $($ male $=1)$ & 0.012 & 0.9398 & & \\
\hline$A o-B N P$ & 0.196 & 0.2146 & & \\
\hline$C S-B N P$ & 0.321 & 0.0381 & & \\
\hline$(C S-A o) B N P$ & 0.357 & 0.0203 & & \\
\hline$A o-N E$ & 0.327 & 0.0348 & & \\
\hline$C S-N E$ & 0.472 & 0.0016 & & \\
\hline$(C S-A o) N E$ & 0.481 & 0.0013 & 0.481 & 0.0013 \\
\hline$L V E F$ & -0.371 & 0.0156 & & \\
\hline PCWP & 0.270 & 0.0835 & & \\
\hline$C T R$ & 0.178 & 0.2602 & & \\
\hline
\end{tabular}

Abbreviations as in Table 2.
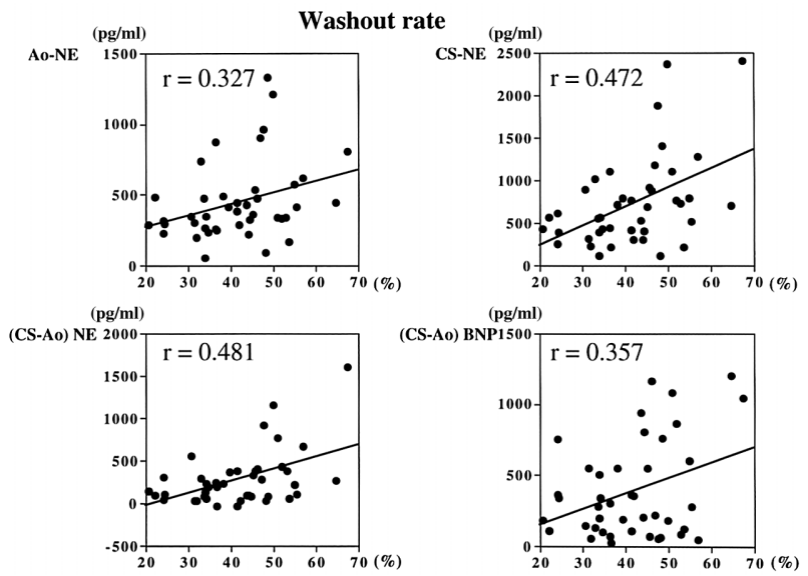

Fig 3. Correlations between washout rate and neurohumoral factors in patients with dilated cardiomyopathy.

washout rate in patients with DCM.

\section{Discussion}

Although the parameters of MIBG provide important prognostic information in $\mathrm{CHF}$ patients with DCM, the precise mechanism that regulates these parameters remains unknown, especially in CHF. We demonstrated (1) a significant and independent correlation of the $\mathrm{H} / \mathrm{M}$ ratio (delayed) and the transcardiac gradient of BNP, suggesting that the $\mathrm{H} / \mathrm{M}$ ratio reflects myocardial dysfunction and/or damage, and (2) a significant and independent correlation of washout rate and the transcardiac gradient of NE, suggesting that the washout rate may reflect an altered cardiac sympathetic nerve terminal in CHF patients with DCM.

\section{Transcardiac Difference of BNP and NE in Patients With $\mathrm{CHF}$}

In the present study, both the BNP concentration and NE concentration showed a significant step-up from the Ao to the CS. We consider that in the present patients, NE spillover from the myocardium was increased and BNP secretion from the ventricle was increased. BNP is mainly secreted from ventricular myocytes and increases with the severity of $\mathrm{CHF}, 17,18,23$ therefore, our data are consistent with those of previous studies. However, it is difficult to assess NE kinetics in the heart, because NE is released, taken back up and metabolized in synaptic fibers ${ }^{24-28} \mathrm{We}$ measured plasma levels of NE in both the Ao and CS, and found a significant increase of NE throughout the heart and compared this transcardiac difference of NE with the parameters of MIBG. NE spillover in the heart can be precisely evaluated by a kinetic study using tritiated $\mathrm{NE}^{26}$ but we did not perform such an evaluation in the present study. To our knowledge, there are no reports indicating whether the transcardiac gradient of NE is reflected by NE spillover in the heart and it remains controversial which mechanisms mainly affect the increased spillover of NE from the myocardium. $2^{2-31}$ However, it has been reported that the transcardiac gradient of NE is more useful for evaluating $\mathrm{CHF}$ than the peripheral concentration of NE$?^{2}$

\section{Relationships Between Cardiac MIBG and Hemodynamic Parameters}

Sympathetic hyperactivity is one of the main compensatory mechanisms of CHF. As heart failure progresses, the cardiac stores of $\mathrm{NE}$ are depleted and the circulating $\mathrm{NE}$ concentration increases! ${ }^{2,33-35}$ The cardiac MIBG image can estimate cardiac sympathetic activity specifically and noninvasively. Merlet et al reported the correlation between H/M(delayed) of cardiac MIBG and LVEF, cardiac output and left ventricular end-diastolic pressure in patients with $\mathrm{CHF}, 10$ and moreover, Imamura et al reported that the washout rate correlates with cardiac index and peak filling rate 36 The present study showed that MIBG parameters 
were weakly, but significantly correlated with LVEF and PCWP, which is consistent with the previous studies.

\section{Relationship Between Cardiac MIBG and \\ the Transcardiac Gradient of NE and \\ $B N P$ in Patients With $\mathrm{CHF}$}

Both cardiac MIBG and neurohumoral factors, such as $\mathrm{NE}$ and BNP, have been shown to be strong and important prognostic indicators in CHF, but few studies have reported the relationships between the MIBG parameters and neurohumoral factors ${ }^{17,18,20}$ In addition, the relationship between the transcardiac gradient of NE and BNP and the H/M ratio and washout rate has not been evaluated in CHF patients with DCM. In the present study, according to stepwise multivariate analysis, the transcardiac gradient of BNP was an independent predictor of the $\mathrm{H} / \mathrm{M}$ (delayed) ratio. It is well known that BNP is a cardiac hormone that acts against ventricular overload from stressors such as myocardial ischemia, necrosis, damage and local mechanical stress on ventricular myocytes 37 Moreover, BNP is superior to other neurohumoral factors as a predictor of prognosis in CHF! ${ }^{17,18,20}$ However, there is no doubt that MIBG uptake reflects sympathetic nervous activity; a previous study reported that MIBG uptake activity significantly correlated with myocardial NE concentrations derived from endomyocardial biopsy samples 38 The H/M ratio is usually assessed as an index of the global uptake of MIBG. Heart size may affect the $\mathrm{H} / \mathrm{M}$ ratio, because the ratio uses the mean counts per heart region, but in the present study there was no correlation between the H/M ratio and CTR. Therefore, we consider that the $\mathrm{H} / \mathrm{M}$ ratio reflects not only a component of sympathetic nervous function, but also myocardial dysfunction and damage in CHF patients with DCM.

In the present study, we recognized that the transcardiac gradient of the NE was an independent predictor of the MIBG washout rate. The transcardiac gradient of NE is supposed to be associated with NE spillover, reflecting sympathetic nervous activity. However, measurement of NE spillover from the heart requires catheterization of the CS or establishment of an equilibrium between endogenous $\mathrm{NE}$ and infused tritiated NE. Our report suggests that the washout rate is a useful tool for evaluating cardiac sympathetic nervous function, and the washout rate can assess altered sympathetic function easily and be repeatedly measured during disease progression. By assessing serial changes on MIBG images, it may be possible to predict worsening of the cardiac sympathetic nervous activity.

\section{Study Limitations}

The present study had several potential weaknesses that could have influenced the results. We did not measure CS flow, but as we were evaluating DCM patients, patients with a significant coronary stenosis were excluded, indicating that the transcardiac gradient of NE and BNP can reflect the total amount of NE and BNP spillover of the heart. Moreover, the NE spillover may not be affected by flow, as the CS flow is slower than that of other organs? ${ }^{4}$ Gilbert et al also reported an evaluation of the adrenergic activity of chronic treatment with different types $\beta$-blockers using the transcardiac gradient of NE without measurement of coronary blood flow? 32

Another potential problem is that treatments varied among the patients and we did not clarify the effects of their medications on cardiac MIBG activity. However, none of the patients were using medications that directly interfere with cardiac MIBG uptake, such as tricyclic antidepressants, and a recent study suggested that $\beta$-blocker therapy does not directly influence MIBG uptake? It will be necessary to investigate whether other medications interfere with MIBG imaging in a larger numbers of patients in the future.

Finally, we did not assess the H/M ratio (early), because that ratio may not reflect neuronal uptake precisely; a previous report demonstrated that early myocardial uptake between DCM patients and control subjects did not significantly differ 39

\section{Conclusion}

The H/M(delayed) of MIBG reflects not only sympathetic nervous activity, but also myocardial dysfunction or injury (transcardiac gradient of BNP) in patients with DCM. The washout rate of MIBG reflects NE spillover from the heart, suggesting a cardiac sympathetic nervous abnormality. Both the $\mathrm{H} / \mathrm{M}$ ratio and washout rate are noninvasive means of providing important information about the failing ventricle of DCM.

\section{Acknowledgments}

We thank Dr Shinro Matsuo, Dr Junya Kusukawa and the nuclear medical staff at the Shiga University of Medical Science. We also wish to thank Ms Ikuko Sakaguchi for her excellent technical assistance.

\section{References}

1. Sisson JC, Wieland DM, Sheman P, Mangner TJ, Tobes MC, Jacques S Jr: Metaiodobenzylguanidine as an index of the adrenergic nervous system integrity and function. J Nucl Med 1987; 28: 1620-1624

2. Wieland DM, Brown LE, Les Roger W, Worthington KC, Wu J-I, Clinthorne $\mathrm{NH}$, et al: Myocardial imaging with a radioiodinated norepinephrine storage analog. J Nucl Med 1981; 22: 22-31

3. Merlet P, Valette H, Dubois Rande JL, Moyse D, Duboc D, Dove P, et al: Prognostic value of cardiac MIBG imaging in patients with congestive heart failure. J Nucl Med 1992; 33: 471-477

4. Nakata T, Miyamoto K, Doi A, Sasao H, Wakabayashi T, Kobayashi $\mathrm{H}$, et al: Cardiac death prediction and impaired cardiac sympathetic innervation assessed by MIBG in patients with failing and nonfailing hearts. J Nucl Cardiol 1998; 5: 579-590

5. Cohen-Solal A, Esanu Y, Logeart D, Pessione F, Dubois C, Dreyfus $\mathrm{G}$, et al: Cardiac metaiodobenzylguanidine uptake in patients with moderate chronic heart failure: Relationship with peak oxygen uptake and prognosis. J Am Coll Cardiol 1999; 33: 759-766

6. Momose M, Kobayashi H, Icuchi N, Matsuda N, Sakomura Y, Kasanuki H, et al: Comparison of parameters of ${ }^{123}$ I-MIBG scintigraphy for predicting prognosis in patients with dilated cardiomyopathy. Nucl Med Commun 1999; 20: 529-535

7. Suwa M, Otake Y, Moriguchi A, Ito T, Hirota Y, Kawamura K, et al: Iodine-123 metaiodobenzylguanidine myocardial scintigraphy for prediction of response to beta-blocker therapy in patients with dilated cardiomyopathy. Am Heart J 1997; 133: 353-358

8. Fukuoka S, Hayashida K, Hirose Y, Shimotsu Y, Ishida Y, Kakuchi $\mathrm{H}$, et al: Use of iodine-123 metaiodobenzylguanidine myocardial imaging to predict the effectiveness of beta-blocker therapy in patients with dilated cardiomyopathy. Eur J Nucl Med 1997; 24: 523-529

9. Merlet P, Pouillart F, Dubois-Rande JL, Delahaye N, Fumey R, Castaigne A, et al: Sympathetic nerve alterations assessed with 123IMIBG in failing human heart. J Nucl Med 1999; 40: 224-231

10. Merlet P, Dubois-Rande JL, Adnot S, Bourguignon MH, Benvenuti C, Loisance D, et al: Myocardial beta-adrenergic desensitization and neuronal norepinephrine uptake function in idiopathic dilated cardiomyopathy. J Cardiovascu Pharmacol 1992; 19: 10-16

11. Imamura Y, Ando H, Mitsuoka W, Egashira S, Masayuki H, Ashihara $\mathrm{T}$, et al: Iodine-123 metaiodobenzylguanidine images reflect intense myocardial adrenergic nervous activity in congestive heart failure independent of underlying cause. J Am Coll Cardiol 1995; 26: 1594 1599

12. Cohn JN, Levine TB, Olivari MT, Garberg V, Lura D, Francis GS, et al: Plasma norepinephrine as a guide to prognosis in patients with 
chronic congestive heart failure. $N$ Engl J Med 1984; 311: 819-823

13. Packer M: Neurohormonal interactions and adaptations in congestive heart failure. Circulation 1988; 77: 721-729

14. Tsutamoto T, Hisanaga T, Fukai D, Wada A, Maeda Y, Maeda K, et al: Prognostic value of plasma intercellular adhesion molecule-1 and endothelin- 1 concentration in patients with chronic congestive heart failure. Am J Cardiol 1995; 76: 803-808

15. Gottlieb SS, Kukin MC, Ahern D, Packer M: Prognostic importance of atrial natriuretic peptide in patients with chronic heart failure. $J$ Am Coll Cardiol 1989; 13: 1534-1539

16. Richards AM, Nicholls MG, Yandle TG, Frampton C, Espiner EA, Turner JG, et al: Plasma N-terminal pro-brain natriuretic peptide and adrenomedullin: New neurohormonal predictors of left ventricular function and prognosis after myocardial infarction. Circulation 1998; 97: $1921-1929$

17. Tsutamoto T, Wada A, Maeda K, Hisanaga T, Mabuchi N, Hayashi $\mathrm{M}$, et al: Plasma brain natriuretic peptide level as a biochemical marker of morbidity and mortality in patients with asymptomatic or minimally symptomatic left ventricular dysfunction: Comparison with plasma angiotensin II and endothelin-1. Eur Heart J 1999; 20: $1799-1807$

18. Tsutamoto T, Wada A, Maeda K, Hisanaga T, Maeda Y, Fukai D, et al: Attenuation of compensation of endogenous cardiac natriuretic peptide system in chronic heart failure (prognostic role of plasma brain natriuretic peptide concentration in patients with chronic symptomatic left ventricular dysfunction). Circulation 1997; 96: 509-516

19. Yasue H, Yoshimura M, Sumida H, Kikuta K, Kugiyama K, Jougasaki M, et al: Localization and mechanism of secretion of Btype natriuretic peptide in comparison with those of A-type natriuretic peptide in normal subjects and patients with heart failure. Circulation 1994; 90: 195-203

20. Maeda K, Tsutamoto T, Wada A, Mabuchi N, Hayashi M, Tsutsui T, et al: High levels of plasma brain natriuretic peptide and interleukin6 after optimized treatment for heart failure are independent risk factors for morbidity and mortality in patients with congestive heart failure. J Am Coll Cardiol 2000; 36: 1587-1593

21. Hara Y, Hamada M, Shigematsu Y, Suzuki M, Kodama K, Kuwahara $\mathrm{T}$, et al: Effect of beta-blocker on left ventricular function and natriuretic peptides in patients with chronic heart failure treated with angiotensin-converting enzyme inhibitor. Jpn Circ J 2000; 64: $365-$ 369

22. Mabuchi N, Tsutamoto T, Maeda K, Kinoshita M: Plasma cardiac natriuretic peptides as biochemical markers of recurrence of atrial fibrillation in patients with mild congestive heart failure. Jpn Circ J 2000; 64: 765-771

23. Yoshimura M, Yasue H, Okumura K, Ogawa H, Jougasaki M, Mukoyama M, et al: Different secretion patterns of atrial natriuretic peptide and brain natriuretic peptide in patients with congestive heart failure. Circulation 1993; 87: 464-469

24. Chidsey CA, Braunwald E, Morrow AG, Mason DT: Myocardial norepinephrine concentrations in man: Effects of reserpine and of congestive heart failure. $N$ Engl J Med 1963; 269: 653-659

25. Esler M, Jennings G, Kopner P, Willett I, Dudley F, Hasking G, et al:
Assessment of human sympathetic nervous system activity from measurements of norepinephrine turnover. Hypertension 1988; 11: $3-20$

26. Hasking GJ, Esler MD, Jennings GL, Burton D, Johns JA, Korner PI: Norepinephrine spillover to plasma in patients with congestive heart failure: Evidence of increased overall and cardiorenal sympathetic nervous activity. Circulation 1986; 73: 615-621

27. Esler M: Assessment of sympathetic nervous function in humans from noradrenaline plasma kinetics. Clin Sci 1982; 62: 247-254

28. Esler M, Jennings G, Korner P, Blombery P, Sacharias N, Leonard P: Measurement of total and organ-specific norepinephrine kinetics in humans. Am J Physiol 1984; 247: E21 -E28

29. Sole MJ, Helke CJ, Jacobowitz DM: Increased dopamine in the failing hamster heart: Transvascular transport of dopamine limits the rate of norepinephrine synthesis. Am J Cardiol 1982; 49: 1682-1690

30. Eisenhofer G, Smolich JJ, Cox HS, Esler MD: Neuronal reuptake of norepinephrine and production of dihydroxyphenylglycol by cardiac sympathetic nerves in the anesthetized dog. Circulation 1991; 84: $1354-1363$

31. Bohm M, La Rosee K, Schwinger RH, Erdmann E: Evidence for reduction of norepinephrine uptake sites in the failing human heart. $J$ Am Coll Cardiol 1995; 25: 146-153

32. Gilbert EM, Abraham WT, Olsen S, Hattler B, White M, Mealy P, et al: Comparative hemodyamic, left ventricular functional, and antiadrenergic effects of chronic treatment with metoprolol versus carvedilol in the failing heart. Circulation 1996; 94: 2817-2825

33. Thomas JA, Marks BH: Plasma norepinephrine in congestive heart failure. Am J Cardiol 1978; 41: 233-243

34. Davis D, Baily R, Zelis GJ: Abnormalities in systemic norepinephrine kinetics in human congestive heart failure. Am J Physiol 1988; 254: $760-766$

35. Abraham WT, Hensen J, Schrier RW: Elevated plasma noradrenaline concentrations in patients with low-output cardiac failure: Dependence on increased noradrenaline secretion rates. Clin Sci 1990; 79: 429-435

36. Imamura $\mathrm{Y}$, Ando H, Ashihara T, Fukuyama T: Myocardial adrenergic nervous activity is intensified in patients with heart failure without left ventricular volume or pressure overload. J Am Coll Cardiol 1996; 8: $371-375$

37. Maeda K, Tsutamoto T, Wada A, Hisanaga T, Kinoshita M: Plasma brain natriuretic peptide as a biochemical marker of high left ventricular end-diastolic pressure in patients with symptomatic left ventricular dysfunction. Am Heart J 1998; 135: 825-832

38. Schofer J, Spielmann R, Schuchert A, Weber K, Schluter M: Iodine123-metaiodobenzylguanidine scintigraphy: A noninvasive method to demonstrate myocardial adrenergic nervous system disintegrity in patients with idiopathic dilated cardiomyopathy. J Am Coll Cardiol 1988; 12: $1252-1258$

39. Henderson EB, Kahn JK, Corbett JR, Jansen DE, Pippin JJ, Kulkarni P, et al: Abnormal I-123 metaiodobenzylguanidine myocardial adrenergic derangement in patients with congestive cardiomyopathy. Circulation 1988; 78: 1192-1199 